\title{
Research Article \\ On the Line Graph of the Zero Divisor Graph for the Ring of Gaussian Integers Modulo $n$
}

\author{
Khalida Nazzal ${ }^{1}$ and Manal Ghanem ${ }^{2}$ \\ ${ }^{1}$ Department of Mathematics, Palestine Technical University-Kadoorie, P.O. Box 7 Tulkarm, \\ West Bank, Palestine \\ ${ }^{2}$ Department of Mathematics, Irbid National University, P.O. Box 2600 Irbid, 21110, Jordan \\ Correspondence should be addressed to Manal Ghanem,dr_mghanem@yahoo.com
}

Received 9 September 2011; Accepted 26 December 2011

Academic Editor: Gelasio Salazar

Copyright (C) 2012 K. Nazzal and M. Ghanem. This is an open access article distributed under the Creative Commons Attribution License, which permits unrestricted use, distribution, and reproduction in any medium, provided the original work is properly cited.

Let $\Gamma\left(\mathbb{Z}_{n}[i]\right)$ be the zero divisor graph for the ring of the Gaussian integers modulo $n$. Several properties of the line graph of $\Gamma\left(\mathbb{Z}_{n}[i]\right), L\left(\Gamma\left(\mathbb{Z}_{n}[i]\right)\right)$ are studied. It is determined when $L\left(\Gamma\left(\mathbb{Z}_{n}[i]\right)\right)$ is Eulerian, Hamiltonian, or planer. The girth, the diameter, the radius, and the chromatic and clique numbers of this graph are found. In addition, the domination number of $L\left(\Gamma\left(\mathbb{Z}_{n}[i]\right)\right)$ is given when $n$ is a power of a prime. On the other hand, several graph invariants for $\Gamma\left(\mathbb{Z}_{n}[i]\right)$ are also determined.

\section{Introduction}

The study of zero divisor graphs of commutative rings reveals interesting relations between ring theory and graph theory; algebraic tools help understand graphs properties and vise versa. In 1988, Beck [1] defined the concept of zero divisor graph of a commutative ring $R$, where the vertices of this graph are all elements in the ring and two vertices $x, y$ are adjacent if and only if $x y=0$. Anderson and Livingston [2] modified the definition of zero divisor graphs by restricting the vertices to the nonzero zero divisors of the ring $R$. Further study of zero divisor graphs by Anderson et al. [3] investigated several graph theoretic properties, such as the number of cliques in $\Gamma(R)$. They also gave some cases in which $\Gamma(R)$ is planer. On the other hand, they answered the question when $\Gamma\left(R_{1}\right) \cong \Gamma\left(R_{2}\right)$ for some specified types of rings $R_{1}$ and $R_{2}$. Akbari and Mohammadian [4] improved on those results. $\Gamma(R)$ for rings $R$ which satisfy certain conditions are discussed by Anderson and Badawi [5]. The zero divisor graph of the ring of integers modulo $n$ was extensively studied in [6-10].

In 2008, Abu Osba et al. [11] introduced the zero divisor graphs for the ring of Gaussian integers modulo $n, \Gamma\left(\mathbb{Z}_{n}[i]\right)$, where they studied several graph properties and 
determined several graph invariants for $\Gamma\left(\mathbb{Z}_{n}[i]\right)$. Further properties of the zero divisor graphs for the ring of Gaussian integers modulo $n$ are investigated in [12].

In this paper, we study the line graph of $\Gamma\left(\mathbb{Z}_{n}[i]\right)$. We organized our work as follows: some basic definitions and terminology are given in Section 2. In Sections 3 and 4, we answer the question when is the line graph $L\left(\Gamma\left(\mathbb{Z}_{n}[i]\right)\right)$ Eulerian, Hamiltonian, or planer. In Section 5, the chromatic and clique numbers of $L\left(\Gamma\left(\mathbb{Z}_{n}[i]\right)\right)$ are found. While the diameter, the girth and the radius of $L\left(\Gamma\left(\mathbb{Z}_{n}[i]\right)\right)$ are determined in Sections 6 and 7, respectively. Finally, the last two sections discuss the domination number of $\Gamma\left(\mathbb{Z}_{n}[i]\right)$ and $L\left(\Gamma\left(\mathbb{Z}_{n}[i]\right)\right)$ as well as the independence and clique numbers of $\Gamma\left(\mathbb{Z}_{n}[i]\right)$.

\section{Preliminaries}

The set of Gaussian integers is defined by $\mathbb{Z}[i]=\{a+b i: a, b \in \mathbb{Z}$ and $i=\sqrt{-1}\}$.

A prime Gaussian integer is one of the following:

(i) $(1+i)$ or $(1-i)$,

(ii) $q$, where $q$ is a prime integer and $q \equiv 3(\bmod 4)$,

(iii) $a+b i, a-b i$, where $a^{2}+b^{2}=p, p$ is a prime integer and $p \equiv 1(\bmod 4)$.

It is clear that $\mathbb{Z}_{n}[i]$ is a ring with addition and multiplications modulo $n$. Throughout this paper, $p$ will be used to denote a prime integer which is congruent to 1 modulo 4 , while $q$ will denote a prime integer which is congruent to 3 modulo 4 . Since $\mathbb{Z}_{n}[i]$ is finite, each element in $\mathbb{Z}_{n}[i]$ is either a zero divisor or a unit. Also, since $\mathbb{Z}[i]$ is a unique factorization domain, each integer $n$ can be uniquely factorized as $n=\prod_{j=1}^{k} \pi_{j}^{m_{j}}$ where $\pi_{j}^{\prime}$ s are Gaussian prime integers and $m_{j}^{\prime} s$ are positive integers.

The zero divisor graph of a commutative ring $R$ denoted by $\Gamma(R)$, is the graph whose vertices is the set of all nonzero zero divisors of $R$, and edge set $E(\Gamma(R))=\{x y: x, y \in$ $V(\Gamma(R))$ and $x y=0\}$. The line graph $L(G)$ of a graph $G$ is defined to be the graph whose vertices are the edges of $G$, with two vertices being adjacent if the corresponding edges share a vertex in $G$. For $\Gamma\left(\mathbb{Z}_{n}[i]\right)$, if $n=2$, then this graph is one vertex, while if $n=q$, then $\Gamma\left(\mathbb{Z}_{n}[i]\right)=$ $K_{0}$. Throughout this paper, all rings, $R$, are commutative with unity.

For a connected graph $G$, the distance, $d(u, v)$, between two vertices $u$ and $v$ is the minimum of the lengths of all $u-v$ paths of $G$. The eccentricity of a vertex $v$ in $G$ is the maximum distance from $v$ to any vertex in $G$. The radius of $G, \operatorname{rad}(G)$, is the minimum eccentricity among the vertices of $G$. The diameter of $G, \operatorname{diam}(G)$, is the maximum eccentricity among the vertices of $G$. The girth of $G, g(G)$, is the length of a shortest cycle in $G$. The center of $G$ is the set of all vertices of $G$ with eccentricity equal to the radius. If $G$ has a walk that traverses each edge exactly once goes through all vertices and ends at the starting vertex, then $G$ is called Eulerian. A graph is called Hamiltonian if there exists a cycle containing every vertex. The chromatic number of a graph $G, \chi(G)$, is the minimum $k$ such that $G$ is $k$-colorable (i.e., can be colored using $k$ different colors such that no two adjacent vertices have the same color). The clique number, $\omega(G)$, of a graph $G$ is the maximum order among the complete subgraphs of $G$. A subset $D$ of the vertex set $V(G)$ is said to be independent if no two vertices in this set are adjacent. The independence number of $G, \beta(G)$, is the maximum cardinality of all independent sets in $G$. A subset $D$ of the vertex set $V(G)$ of a graph $G$ is a dominating set in $G$ if each vertex of $G$, not in $D$, is adjacent to at least one vertex of $D$. The minimum cardinality of all dominating sets in $G, \gamma(G)$, is called the domination number of $G$. Edge dominating sets are defined analogously. The minimum cardinality of all edge dominating sets in $G, \grave{\gamma}(G)$, is called the edge domination number of $G$. The minimum cardinality of all independent 
edge dominating sets, $\hat{\gamma}_{i}(G)$, is called the independence edge domination number of $G$. The maximum vertex degree of a graph $G$ will be denoted by $\Delta(G)$.

\section{When Is $L\left(\Gamma\left(\mathbb{Z}_{n}[i]\right)\right)$ Eulerian?}

Now it is characterized when the line graph $L\left(\Gamma\left(\mathbb{Z}_{n}[i]\right)\right)$ is Eulerian. Before proceeding, we prove the following lemma.

Lemma 3.1. (i) Every vertex of $\Gamma\left(\mathbb{Z}_{n}[i]\right)$ has even degree if and only if $n=2, p$ or $n$ is a composite integer which is a product of distinct odd primes.

(ii) If $n=t^{m}, m \geq 2$ and $n \neq q^{2}$, then $\Gamma\left(\mathbb{Z}_{n}[i]\right)$ has a vertex of odd degree and another of even degree.

(iii) Every vertex of $\Gamma\left(\mathbb{Z}_{n}[i]\right)$ has odd degree if and only if $n=q^{2}$.

Proof. (i) Since the graph $G$ is Eulerian if and only if each vertex has an even degree by Theorem 29 of [11], the result holds.

(ii) Assume that $n=t^{m}, t$ is prime, $m \geq 2$ and $n \neq q^{2}$. Then we have three cases.

Case $I(t=2)$. Then $\operatorname{deg}(1+i)=1$ and $\operatorname{deg}\left(2^{m-1}+2^{m-1} i\right)=2^{2 m-1}-2$.

Case II ( $t$ is an odd prime and $m>2)$. By Theorem 23 of [11], $\Gamma\left(\mathbb{Z}_{n}[i]\right)$ has a vertex of degree $t^{2 k-1}-1$, where $1 \leq k<m / 2$ and a vertex of degree $t^{2 k}-2$, where $m / 2 \leq k<m$.

Case III $\left(t=p=a^{2}+b^{2}\right.$ and $\left.m=2\right)$. Since $\operatorname{deg}(a+i b)=|\langle p(a-i b)\rangle|-1$ and $|\langle p(a-i b)\rangle|$ divides $\left|\mathbb{Z}_{p^{2}}\right|,|\langle p(a-i b)\rangle|$ is odd and hence $\operatorname{deg}(a+i b)$ is even. Then by using part (i), the result holds.

(iii) $(\Rightarrow)$ Let $n=\prod_{j=1}^{k} a_{j}^{m_{j}}, k \geq 2$, and $\bar{x}_{j}=\left(x_{t}\right)$, where

$$
x_{t}= \begin{cases}1, & \text { if } t=j, \\ 0, & \text { otherwise }\end{cases}
$$

Now if all $a_{j}^{\prime} s$ are odd primes, then $\operatorname{deg}\left(\bar{x}_{j}\right)=n /\left(a_{j}^{m_{j}}\right)-1$ and if $a_{1}=2$, then $\operatorname{deg}\left(\bar{x}_{1}\right)=$ $\left(n / 2^{m_{1}}\right)-1$.

$(\Leftarrow)$ Note that, $\Gamma\left(\mathbb{Z}_{q^{2}}[i]\right) \cong K_{q^{2}-1}$. So, $\operatorname{deg}(v)=q^{2}-2$ for every vertex $v$ in $\Gamma\left(\mathbb{Z}_{q^{2}}[i]\right)$.

Since $L(\Gamma(R))$ is Eulerian if and only if $\operatorname{deg}(v)$ is even for every $v \in \Gamma(R)$ or $\operatorname{deg}(v)$ is odd for every $v \in \Gamma(R)$, [9], together with Lemma 3.1 and Theorem 26 of [11], the following theorem is obtained.

Theorem 3.2. (i) $L\left(\Gamma\left(\mathbb{Z}_{n}[i]\right)\right)$ is Eulerian graph if and only if $n=2, p, q^{2}$, or $n$ is a composite integer which is a product of distinct odd primes.

(ii) $L\left(\Gamma\left(\mathbb{Z}_{n}[i]\right)\right)$ is Eulerian graph does not necessarily imply that $\Gamma\left(\mathbb{Z}_{n}[i]\right)$ is Eulerian.

\section{When Is $L\left(\Gamma\left(\mathbb{Z}_{n}[i]\right)\right)$ Hamiltonian or Planner?}

First we determine which graphs, $\Gamma\left(\mathbb{Z}_{n}[i]\right)$, are Hamiltonian. Before this paper comes to the light, a recent article by Abu Osba et al. [12] reached to similar results concerning 
Hamiltonian $\Gamma\left(\mathbb{Z}_{n}[i]\right)$. However, we present our proof since it is simpler and shorter. The proof makes use of the following theorem.

Theorem 4.1 (see [4]). Let $R$ be a finite principal ideal ring, if $\Gamma(R)$ is Hamiltonian, then it is either a complete graph or a complete bipartite graph.

Theorem 4.2. The graph $\Gamma\left(\mathbb{Z}_{n}[i]\right)$ is Hamiltonian if and only if $n=p$ or $q^{2}$.

Proof. Since $\mathbb{Z}_{n}[i]$ is a finite principal ideal ring, $\Gamma\left(\mathbb{Z}_{n}[i]\right)$ is a complete graph or a complete bipartite graph if $\Gamma\left(\mathbb{Z}_{n}[i]\right)$ is Hamiltonian. But the graph $\Gamma\left(\mathbb{Z}_{n}[i]\right)$ is complete if and only if $n=q^{2}$, and it is complete bipartite if and only if $n=p$ or $q_{1} q_{2}$, where $q_{1}<q_{2}$, [11]. On the other hand, a complete bipartite graph $K_{m, n}$ is Hamiltonian if and only if $m=n$. So the result holds.

Note that $\Gamma\left(\mathbb{Z}_{q_{1} q_{2}}[i]\right)$ is not Hamiltonian and hence the converse of Theorem 4.1 is not true.

Next, we move to the line graphs $L\left(\Gamma\left(\mathbb{Z}_{n}[i]\right)\right)$. Before proceeding, we present the following theorem.

Theorem 4.3. (i) If $G$ is a graph of diameter at most 2 with $|V(G)| \geq 4$, then $L(G)$ is Hamiltonian, see [13].

(ii) The line graph of an Eulerian graph is both Hamiltonian and Eulerian, see [14].

If $n=p, 2^{m}$, or $q^{m}$, where $m \geq 2$, then diam $\left(\Gamma\left(\mathbb{Z}_{n}[i]\right)\right) \leq 2$. On the other hand, if $n=2, p$ or $n$ is a composite odd integer which is a product of distinct primes, then $\Gamma\left(\mathbb{Z}_{n}[i]\right)$ is Eulerian, [11]. Thus the following corollary is obtained.

Corollary 4.4. (i) If $n=p, 2^{m}$, or $q^{m}$, where $m \geq 2$, then $L\left(\Gamma\left(\mathbb{Z}_{n}[i]\right)\right)$ is Hamiltonian.

(ii) If $n$ is a composite odd integer which is a product of distinct primes, then $L\left(\Gamma\left(\mathbb{Z}_{n}[i]\right)\right)$ is both Eulerian and Hamiltonian.

Now, we discuss planarity of the graph $L\left(\Gamma\left(\mathbb{Z}_{n}[i]\right)\right)$.

A graph $G$ is planar if it can be drawn in the plane without any edge crossing. The following theorem gives necessary and sufficient conditions on a graph $G$ so that the line graph $L(G)$ is planer.

Theorem 4.5 (see [15]). A nonempty graph $G$ has a planer line graph $L(G)$ if and only if

(i) $G$ is planer,

(ii) $\Delta(G) \leq 4$,

(iii) if $\operatorname{deg}_{G}(v)=4$, then $v$ is a cut vertex.

Recall that $\Gamma\left(\mathbb{Z}_{n}[i]\right)$ is planer if and only if $n=2$ or $n=4$, [11]. But $L\left(\Gamma\left(\mathbb{Z}_{4}[i]\right)\right)$ is not planer since $\Delta\left(\Gamma\left(\mathbb{Z}_{4}[i]\right)\right)=7>4$. Therefore, we get the following theorem.

Theorem 4.6. The graph $L\left(\Gamma\left(\mathbb{Z}_{n}[i]\right)\right)$ is never planer. 


\section{The Chromatic and Clique Numbers of $L\left(\Gamma\left(\mathbb{Z}_{n}[i]\right)\right)$}

If $R$ is a finite ring, then $\chi^{\prime}(\Gamma(R))=\Delta(\Gamma(R))$, unless $\Gamma(R)$ is complete graph of odd order, [4]. Note that, the only complete graph $\Gamma\left(\mathbb{Z}_{n}[i]\right)$ occurs when $n=q^{2}$. However, in this case the order of the graph is $q^{2}-1$ which is even, so $x^{\prime}\left(\Gamma\left(\mathbb{Z}_{n}[i]\right)\right)=\Delta\left(\Gamma\left(\mathbb{Z}_{n}[i]\right)\right)$. Moreover, since the edge coloring of any graph leads to a vertex coloring of its line graph, we obtain $\chi\left(L\left(\Gamma\left(\mathbb{Z}_{n}[i]\right)\right)\right)=\Delta\left(\Gamma\left(\mathbb{Z}_{n}[i]\right)\right)$. Clearly, $\chi(G) \geq \omega(G)$. On the other hand, the line graph of $G$ has a complete subgraph of order $\Delta(G)$. Thus $\omega\left(L\left(\Gamma\left(\mathbb{Z}_{n}[i]\right)\right)\right) \geq \Delta\left(\Gamma\left(\mathbb{Z}_{n}[i]\right)\right)$. Observe that if $n=2^{m}$ or $q^{m}, m \geq 2$, then $\Gamma\left(\mathbb{Z}_{n}[i]\right)$ has a vertex which is adjacent to every other vertex in $\Gamma\left(\mathbb{Z}_{n}[i]\right)$. While if $n=p^{m}, m \geq 1$, then $\mathbb{Z}_{p^{m}}[i] \cong \mathbb{Z}_{p^{m}} \times \mathbb{Z}_{p^{m}}$. Thus $\Delta\left(\Gamma\left(\mathbb{Z}_{p^{m}}[i]\right)\right)=p^{2 m-1}-1$. This leads to the following theorem.

\section{Theorem 5.1.}

$$
\omega\left(L\left(\Gamma\left(\mathbb{Z}_{n}[i]\right)\right)\right)=\chi\left(L\left(\Gamma\left(\mathbb{Z}_{n}[i]\right)\right)\right)= \begin{cases}2^{2 m-1}-2, & \text { if } n=2^{m}, m \geq 2, \\ q^{2 m-2}-2, & \text { if } n=q^{m}, m \geq 2, \\ p^{2 m-1}-1, & \text { if } n=p^{m}, m \geq 1 .\end{cases}
$$

Finally, if $n=2^{m} \prod_{j=1}^{r} p_{j}^{r_{j}} \prod_{j=1}^{l} q_{j} \prod_{j=1}^{s} q_{j}^{s_{j}}$, where $s_{j} \geq 2$ and $m, r_{j} \geq 1$, then the clique number and the chromatic number for the graph $L\left(\Gamma\left(\mathbb{Z}_{n}[i]\right)\right)$ is given by the following theorem.

Theorem 5.2. $n=2^{m} \prod_{j=1}^{r} p_{j}^{r_{j}} \prod_{j=1}^{l} q_{j} \prod_{j=1}^{s} q_{j}^{s_{j}}$, where $m, r_{j} \geq 1$ and $s_{j} \geq 2$, then

$$
\omega\left(L\left(\Gamma\left(\mathbb{Z}_{n}[i]\right)\right)\right)=\chi\left(L\left(\Gamma\left(\mathbb{Z}_{n}[i]\right)\right)\right)=\left(2^{2 m-1}-1\right) \prod_{j=1}^{r}\left(p_{j}^{2 r_{j-1}}\right) \prod_{j=1}^{s}\left(q_{j}^{2 s_{j}-2}-1\right)-1
$$

Proof. The result follows by computing $\Delta\left(\Gamma\left(\mathbb{Z}_{n}[i]\right)\right)$, since $\Delta\left(\Gamma\left(\mathbb{Z}_{n}[i]\right)\right)=\omega\left(L\left(\Gamma\left(\mathbb{Z}_{n}[i]\right)\right)\right)=$ $x\left(L\left(\Gamma\left(\mathbb{Z}_{n}[i]\right)\right)\right)$.

\section{The Diameter of $L\left(\Gamma\left(\mathbb{Z}_{n}[i]\right)\right)$}

Now, we will find the diameter of the line graph $L\left(\Gamma\left(\mathbb{Z}_{n}[i]\right)\right)$.

First, we will prove that diam $\left(L\left(\Gamma\left(\mathbb{Z}_{n}[i]\right)\right)\right)=2$ when $n=2^{m}$ or $n=q^{m}$.

Lemma 6.1. (i) If $n=2^{m}, m \geq 2$, then there are no $a+b i, c+d i \in \mathbb{Z}_{n}[i]$, where $a, b, c, d$ are odd integers such that $(a+b i)(c+d i) \equiv 0(\bmod 4)$.

(ii) If $n=q^{m}, m \geq 2$ then there are no $a+b i, c+d i \in \mathbb{Z}_{n}[i]$ where $a, b, c, d$ are relatively prime with $q$, such that $(a+b i)(c+d i) \equiv 0(\bmod q)$.

Proof. (i) Assume that $(a+b i)(c+d i) \equiv 0(\bmod 4)$. Then $a c-b d \equiv 0(\bmod 4)$ and $a d+b c \equiv$ $0(\bmod 4)$. Since $a, b, c, d$ are odd integers, $a=2 a_{1}+1, b=2 b_{1}+1, c=2 c_{1}+1$, and $d=2 d_{1}+1$ for some $a_{1}, b_{1}, c_{1}, d_{1} \in \mathbb{Z}$. So $a c-b d \equiv a_{1}+c_{1}+b_{1}+d_{1} \equiv 0(\bmod 2)$. And $a d+b c \equiv a_{1}+c_{1}+b_{1}+$ $d_{1} \equiv 1(\bmod 2)$, a contradiction. 
(ii) Assume that $(a+b i)(c+d i) \equiv 0(\bmod q)$. Then $a c-b d \equiv 0(\bmod q)$ and $a d+b c \equiv$ $0(\bmod q)$. Since $a, b, c, d$ are relatively prime with $q$, we have $a=q a_{1}+a_{2}, b=q b_{1}+b_{2}, c=$ $q c_{1}+c_{2}$ and $d=q d_{1}+d_{2}$, where $0<a_{2}, b_{2}, c_{2}, d_{2}<q$. So

$$
\begin{aligned}
& a c-b d \equiv a_{2} c_{2}-b_{2} d_{2} \equiv 0(\bmod q) \\
& a d+b c \equiv a_{2} d_{2}+b_{2} c_{2} \equiv 0(\bmod q) .
\end{aligned}
$$

Multiplying (6.1) by $c_{2}$ and (6.2) by $d_{2}$ and adding gives $a_{2}\left(c_{2}{ }^{2}+d_{2}{ }^{2}\right) \equiv 0(\bmod q)$. Then $q \mid a_{2}$ or $q \mid\left(c_{2}{ }^{2}+d_{2}{ }^{2}\right)$. Since $a_{2}<q, q \mid\left(c_{2}{ }^{2}+d_{2}{ }^{2}\right)$. Therefore, $c_{2}{ }^{2}+d_{2}{ }^{2} \equiv 0(\bmod q)$, and hence $c_{2} \equiv d_{2} \equiv$ $0(\bmod q)$, a contradiction.

So, we conclude the following.

Theorem 6.2. If $n=2^{m}$ or $q^{m}$ and $m \geq 2$, then $\operatorname{diam}\left(L\left(\Gamma\left(\mathbb{Z}_{n}[i]\right)\right)\right)=2$.

Proof. (i) Suppose that $n=2^{m}, m \geq 2$. Then,

(1) $x=a 2^{t}+b 2^{k} i$ where $a, b$ are odd and $t \neq k$ or $t=k \geq[m / 2]$ implies that ann $(x)=$ $\left\{c 2^{r}+d 2^{s} i: c\right.$ and $d$ are odd and $\left.r, s \geq m-\min \{t, k\}\right\}$,

(2) $x=2^{t}(a+b i)$ where $a, b$ are odd and $t<\lceil m / 2\rceil$, then ann $(x)=\left\{c 2^{r}+d 2^{s} i: c\right.$ and $d$ are odd and $r, s \geq m-t\} \cup\left\{2^{m-t-1}(c+d i): c\right.$ and $d$ are odd $\}$.

Moreover, $d\left(\left[2^{t}\left(a_{1}+b_{1} i\right), 2^{m-t-1}\left(c_{1}+d_{1} i\right)\right],\left[2^{s}\left(a_{2}+b_{2} i\right), 2^{m-s-1}\left(c_{2}+d_{2} i\right)\right]\right)=2$ if $t \leq s<$ $\lceil m / 2\rceil$. Since $\left[2^{s}\left(a_{2}+b_{2} i\right), 2^{m-t-1}\left(c_{1}+d_{1} i\right)\right] \in V\left(L\left(\Gamma\left(\mathbb{Z}_{n}[i]\right)\right)\right)$.

(ii) Suppose that $n=q^{m}, m \geq 2$. Let $x=a q^{t}+b q^{k} i$ and $a, b \in U\left(\mathbb{Z}_{n}\right)$. Then ann $(x)=$ $\left\{c q^{r}+d q^{s} i: r, s \geq m-\min \{t, k\}\right\}$. Moreover, $d\left(\left[a_{1} q^{r_{1}}+b_{1} q^{s_{1}} i, c_{1} q^{t_{1}}+d_{1} q^{k_{1}} i\right],\left[a_{2} q^{r_{2}}+b_{2} q^{s_{2}} i\right.\right.$, $\left.\left.c_{2} q^{t_{2}}+d_{2} q^{k_{2}} i\right]\right)=2$ since $r_{1}, s_{1}, t_{2}, k_{2} \geq[m / 2]$ implies that $\left[a_{1} q^{r_{1}}+b_{1} q^{s_{1}} i, c_{2} q^{t_{2}}+d_{2} q^{k_{2}} i\right] \in$ $V\left(L\left(\Gamma\left(\mathbb{Z}_{n}[i]\right)\right)\right)$.

From Theorems 3.1 and 3.3 of $[9], 2 \leq \operatorname{diam}\left(L\left(\Gamma\left(\mathbb{Z}_{n}[i]\right)\right)\right) \leq 3$. In $L\left(\Gamma\left(\mathbb{Z}_{p^{m}}[i]\right)\right)$, where $p=a^{2}+b^{2}$ and $m \geq 2, d\left(\left[p, p^{m-1}\right],\left[(a+i b)^{m},(a-i b)^{m}\right]\right)=3$. So, $\operatorname{diam}\left(L\left(\Gamma\left(\mathbb{Z}_{p^{m}}[i]\right)\right)\right)=3$.

Theorem 6.3. (i) If $n=s t$, where $s, t$ are two distinct primes and $s \neq p$ or $t \neq p$, then $\operatorname{diam}\left(L\left(\Gamma\left(\mathbb{Z}_{n}[i]\right)\right)\right)=2$.

(ii) If $n=s t^{2}$ are two distinct primes and $s, t \neq p$, then diam $\left(L\left(\Gamma\left(\mathbb{Z}_{n}[i]\right)\right)\right)=2$.

Proof. First note that $\operatorname{diam}(L(\Gamma(R))) \geq 2$, [9] and for $n=n_{1} n_{2}$ with g.c.d $\left(n_{1}, n_{2}\right)=1, \mathbb{Z}_{n}[i] \cong$ $\mathbb{Z}_{n_{1}}[i] \times \mathbb{Z}_{n_{2}}[i]$.

(i) Case I: If $n=q p$ or $n=2 p$ where $p=a^{2}+b^{2}$, then $V\left(L\left(\Gamma\left(\mathbb{Z}_{n}[i]\right)\right)\right)=\{[(u, \alpha(a+$ $b i)),(0, \beta(a-b i))]\} \cup\{[(0, \alpha(a+b i)),(u, \beta(a-b i))]\} \cup\{[(u, 0),(0, v)]\}$.

Case II: If $n=2 q$ or $n=q_{1} q_{2}$, then

$$
V\left(L\left(\Gamma\left(\mathbb{Z}_{n}[i]\right)\right)\right)=\{[(u, 0),(0, v)]: u, v \neq 0\}
$$

(ii) Note that $V\left(L\left(\Gamma\left(\mathbb{Z}_{n}[i]\right)\right)\right)=\{[(u, \alpha t),(0, \beta t)]\} \cup\{[(u, 0),(0, v)]: u, v, \alpha, \beta \neq 0\}$. 
Theorem 6.4. (i) If $n=s p^{2}$, where $s$ is prime and $p=a^{2}+b^{2}$, then $\operatorname{diam}\left(L\left(\Gamma\left(\mathbb{Z}_{n}[i]\right)\right)\right)=3$.

(ii) If $n=p_{1}^{m} p_{2}^{l}$, where $p_{1}=a_{1}^{2}+b_{1}^{2}, p_{2}=a_{2}^{2}+b_{2}^{2}$ and $m, l \geq 1$, then $\operatorname{diam}\left(L\left(\Gamma\left(\mathbb{Z}_{n}[i]\right)\right)\right)=3$.

(iii) If $n=p^{m} t^{l}$, where $p=a^{2}+b^{2}, m \geq 1, l \geq 2$, and g.c.d $(p, t)=1$, then $\operatorname{diam}\left(L\left(\Gamma\left(\mathbb{Z}_{n}[i]\right)\right)\right)=3$.

(iv) If $n=s^{m} t^{l}$ where $s$, $t$ are distinct primes and $m, l \geq 2$, then $\operatorname{diam}\left(L\left(\Gamma\left(\mathbb{Z}_{n}[i]\right)\right)\right)=3$.

Proof. (i) Let $v_{1}=\left[\left(0,(a+b i)^{2}\right),\left(1,(a-b i)^{2}\right)\right]$ and $v_{2}=[(0,(a-b i)(a+b i)),(1,(a-b i)(a+b i))]$. Then $d\left(v_{1}, v_{2}\right)=3$.

(ii) Let $v=\left[\left(\left(a_{1}+b_{1} i\right)^{m},\left(a_{2}+b_{2} i\right)^{l}\right),\left(\left(a_{1}-b_{1} i\right)^{m},\left(a_{2}-b_{2} i\right)^{l}\right)\right]$. Then $d(v,[(1,0),(0,1)])=3$.

(iii) Let $v=\left[\left((a+b i)^{m}, t\right),\left((a-b i)^{m}, t^{l-1}\right)\right]$. Then $d(v,[(1,0),(0,1)])=3$.

(iv) Let $v=\left[(s, t),\left(s^{m-1}, t^{l-1}\right)\right]$. Then $d(v,[(1,0),(0,1)])=3$.

Theorem 6.5. (i) If $R_{1}, R_{2}, R_{3}$ are fields and $R=R_{1} \times R_{2} \times R_{3}$, then diam $(L(\Gamma(R)))=2$.

(ii) If $R_{1}, R_{2}, R_{3}$ are finite rings and $R_{i}$ is not a field for some $i \in\{1,2,3\}$ and $R=R_{1} \times R_{2} \times R_{3}$, then diam $(L(\Gamma(R)))=3$.

(iii) If $R=\Pi_{i=1}^{k} R_{i}$ where $k \geq 4$, then diam $(L(\Gamma(R)))=3$.

Proof. (i) Let $\left[\left(a_{1}, a_{2}, a_{3}\right),\left(b_{1}, b_{2}, b_{3}\right)\right]\left[\left(c_{1}, c_{2}, c_{3}\right),\left(d_{1}, d_{2}, d_{3}\right)\right] \in E(L(\Gamma(R)))$. Since $R_{1}, R_{2}, R_{3}$ are fields, $\left(a_{1}, a_{2}, a_{3}\right)$ or $\left(b_{1}, b_{2}, b_{3}\right)$ has exactly two components equal 0. W.L.O.G. let $\left(a_{1}, a_{2}, a_{3}\right)=$ $\left(a_{1}, 0,0\right)$ and $a_{1} \neq 0$. Since $c_{1} d_{1}=0, c_{1}=0$ or $d_{1}=0$. Say $c_{1}=0$, then $\left[\left(a_{1}, a_{2}, a_{3}\right),\left(c_{1}, c_{2}, c_{3}\right)\right] \in$ $E(L(\Gamma(R)))$. So, $\operatorname{diam}(L(\Gamma(R)))=2$.

(ii) Suppose that $R_{1}$ is not a field. Let $x, y \in R_{1}^{*}$ such that $x y=0$. Then $d([(x, 0,1)$, $(y, 1,0)],[(0,1,1),(1,0,0)])=3$.

(iii) Let $x=\left(x_{j}\right)$, where $x_{j}=1$ if $j=1,2$ and 0 otherwise, $y=\left(y_{j}\right)$, where $y_{j}=1$ if $j=$ 3,4 and 0 otherwise, $z=\left(z_{j}\right)$, where $z_{j}=1$ if $j=2,3$ and 0 otherwise and $w=\left(w_{j}\right)$, where $w_{j}=1$ if $j=1,4$ and 0 otherwise. Then $d([x, y],[z, w])=3$.

Summarizing the above results, we get the following theorem.

Theorem 6.6. (i) $\operatorname{diam}\left(L\left(\Gamma\left(\mathbb{Z}_{n}[i]\right)\right)\right)=2$ if and only if $n=p, 2 q, q_{1} q_{2}, q_{1} q_{2} q_{3}, 2 q_{1} q_{2}, 4 q, 2 q^{2}, 2 p, q p$ or $n=2^{m}, q^{m}$ with $m \geq 2$.

(ii) $\operatorname{diam}\left(L\left(\Gamma\left(\mathbb{Z}_{n}[i]\right)\right)\right)=3$ otherwise.

\section{The Girth and the Radius of $L\left(\Gamma\left(\mathbb{Z}_{n}[i]\right)\right)$}

In this section, we give a complete characterization of the girth and the radius of $L\left(\Gamma\left(\mathbb{Z}_{n}[i]\right)\right)$.

Since for any commutative ring $R, L(\Gamma(R))$ is a tree if and only if $\Gamma(R)=K_{2}$ or $K_{1,2}$ [9], $L\left(\Gamma\left(\mathbb{Z}_{n}[i]\right)\right)$ is never a tree. On the other hand, if $L(\Gamma(R))$ contains a cycle, then $g(L(\Gamma(R))) \leq 4$ where equality holds only if $R=\mathbb{Z}_{3} \times \mathbb{Z}_{3}$, [9].

Consequently, the following result holds.

Theorem 7.1. $g\left(L\left(\Gamma\left(\mathbb{Z}_{n}[i]\right)\right)\right)=3$.

Next, we prove that the radius of the line graph $L\left(\Gamma\left(\mathbb{Z}_{n}[i]\right)\right)$ equals 2 .

Since $\operatorname{diam}(L(\Gamma(R))) \leq 3,[9]$ and $\operatorname{rad}(G) \leq \operatorname{diam}(G)$ for any graph $G, \operatorname{rad}(L(\Gamma(R))) \leq 3$.

Lemma 7.2. If there exists a vertex $v \in L\left(\Gamma\left(\mathbb{Z}_{n}[i]\right)\right)$ with eccentricity 2 , then $\operatorname{rad}\left(L\left(\Gamma\left(\mathbb{Z}_{n}[i]\right)\right)=2\right.$

Proof. Note that, $L\left(\Gamma\left(\mathbb{Z}_{n}[i]\right)\right)$ has no spanning star graph, since if $a, b \in V\left(\Gamma\left(\mathbb{Z}_{n}[i]\right)\right)$ such that $a \neq b$ and $a b=0$, then $d([a, b],[a i, b i])>1$. 
Theorem 7.3. If $n=2^{m}, n=q^{m}, m \geq 2$ or $n=p^{m}, m \geq 1$, then $\operatorname{rad}\left(L\left(\Gamma\left(\mathbb{Z}_{n}[i]\right)\right)\right)=2$.

Proof. (1) If $n=2^{m}, m \geq 2$, then $d\left(\left[2^{m-1}+2^{m-1} i, 2\right],[x, y]\right) \leq 2$ for all $[x, y] \in V\left(L\left(\Gamma\left(\mathbb{Z}_{n}[i]\right)\right)\right)$.

(2) If $n=q^{m}, m \geq 2$, then $d\left(\left[q^{m-1}, q\right],[x, y]\right) \leq 2$ for all $[x, y] \in V\left(L\left(\Gamma\left(\mathbb{Z}_{n}[i]\right)\right)\right)$.

(3) If $n=p^{m}, m \geq 1$, then $d\left(\left[(a+b i)^{m}(a-b i)^{m-1},(a-b i)^{m}(a+b i)^{m-1}\right],[x, y]\right) \leq 2$ for all $[x, y] \in V\left(L\left(\Gamma\left(\mathbb{Z}_{n}[i]\right)\right)\right)$.

Theorem 7.4. If $n=r^{m} t$, where $r=2, q$, or $p$ and $m \geq 1$, g.c.d $(r, t)=1$, then $\operatorname{rad}\left(L\left(\Gamma\left(\mathbb{Z}_{n}[i]\right)\right)\right)=2$.

Proof. (1) If $r=2$ or $q$, then $d\left(\left[\left(r^{m-1}, 0\right),(r, 1)\right],[(x, y),(t, s)]\right) \leq 2$ for all $[(x, y),(t, s)] \in$ $V\left(L\left(\Gamma\left(\mathbb{Z}_{n}[i]\right)\right)\right)$.

(2) If $r=p=a^{2}+b^{2}$, then $d\left(\left[\left((a+b i)^{m}(a-b i)^{m-1}, 0\right),\left((a-b i)^{m}(a+b i)^{m-1}, 0\right)\right]\right.$, $[(x, y),(t, s)]) \leq 2$ for all $[(x, y),(t, s)] \in V\left(L\left(\Gamma\left(\mathbb{Z}_{n}[i]\right)\right)\right)$.

Summarizing the above results, we get the following.

Theorem 7.5. The radius of the line graph $L\left(\Gamma\left(\mathbb{Z}_{n}[i]\right)\right)$ equals 2 .

\section{The Domination Number of $\Gamma\left(\mathbb{Z}_{n}[i]\right)$}

Pervious results concerning the domination number of $\Gamma\left(\mathbb{Z}_{n}[i]\right)$ are very restricted; Abu Osba et al. [11] answered the question "when is the domination number 1 or 2?". Here we find the domination number of the graph $\Gamma\left(\mathbb{Z}_{n}[i]\right)$. Two independent proofs reflecting two different viewpoints are given, the first proof depends on ring theory. While the second proof is constructive in the sense that it does not only give the domination number of $\Gamma\left(\mathbb{Z}_{n}[i]\right)$, but also gives a minimum dominating set of this graph. This dominating set, as we will see, reveals to have interesting properties.

Theorem 8.1 (see [16]). Let $R$ be a finite commutative ring with identity that is not an integral domain. If $\Gamma(R)$ is not a star graph, then the domination number equals the number of distinct maximal ideals of $R$.

Theorem 8.2. If $n=\prod_{j=1}^{k} \pi_{j}^{m_{j}}$, where $k \geq 1$ and $\pi_{j}^{\prime}$ s are distinct gaussian prime and $m_{j}^{\prime}$ s are positive integers and $n \neq 2$ or $q$. Then $\gamma\left(\Gamma\left(\mathbb{Z}_{n}[i]\right)\right)=k$, if $n$ is odd, and $\gamma\left(\Gamma\left(\mathbb{Z}_{n}[i]\right)\right)=k-1$, if $n$ is even.

Proof. (I) (1) If $n=2^{m}$, then $\langle 1+i\rangle$ is the unique maximal ideal of $\mathbb{Z}_{n}[i]$.

(2) If $n=q^{m}$, then $\langle q\rangle$ is the unique maximal ideal of $\mathbb{Z}_{n}[i]$.

(3) If $n=p^{m}$ where $p=a^{2}+b^{2}$, then $\langle a+b i\rangle$ and $\langle a-b i\rangle$ are the only distinct maximal ideals of $\mathbb{Z}_{n}[i]$. ideals of $\mathbb{Z}_{n}[i]$.

(4) If $n=\prod_{j=1}^{k} \pi_{j}^{m_{j}}$ is odd then $\left\langle\pi_{j}\right\rangle \times \prod_{t=1, t \neq j}^{k}\left(\mathbb{Z}[i] /\left\langle\pi_{t}^{m_{t}}\right\rangle\right)$ are the only maximal

(5) If $n=\prod_{j=1}^{k} \pi_{j}^{m_{j}}$ is even then $\pi_{1}=(1+i), \pi_{2}=(1-i)$ and $\left\langle\pi_{j}\right\rangle \times \prod_{t=1, t \neq j}^{k}(\mathbb{Z}[i] /$ $\left.\left\langle\pi_{t}^{m_{t}}\right\rangle\right)$ where $j \neq 2$ are the only distinct maximal ideals of $\mathbb{Z}_{n}[i]$. Finally, since $\Gamma\left(\mathbb{Z}_{n}[i]\right)$ is never a star graph [11], the result holds. 
Proof. (II) We have two cases.

Case I: $n$ is odd. Then it is easy to see that $D=\left\{P_{j}=\pi_{1}^{m_{1}} \pi_{2}^{m_{2}} \ldots \pi_{j}^{m_{j}-1} \ldots \pi_{k}^{m_{k}}: 1 \leq j \leq k\right\}$ is a dominating set of $\Gamma\left(\mathbb{Z}_{n}[i]\right)$. To show that $D$ is a minimum dominating set, assume that $D_{1}$ is a minimum dominating set such that there is no $x=s P_{j}$, g.c.d $\left(s, \pi_{j}\right)=1$ belongs to $D_{1}$ for some $1 \leq j \leq k$. Then $T_{j}=\left\{\pi_{j}, 2 \pi_{j}\right\} \subseteq D_{1}$. So, $\left(D_{1}-T_{j}\right) \cup\left\{P_{j}\right\}$ is a dominating set of $\Gamma\left(\mathbb{Z}_{n}[i]\right)$, a contradiction.

Case II: $n$ is even. Then $\pi_{1}=(1+i), \pi_{2}=(1-i)$. Similar to case I, we can see that $D=$ $\left\{P_{j}=\pi_{1}^{m_{1}} \pi_{2}^{m_{2}} \ldots \pi_{j}^{m_{j}-1} \ldots \pi_{k}^{m_{k}}: 1 \leq j \leq k, j \neq 2\right\}$ is a minimum dominating set of $\Gamma\left(\mathbb{Z}_{n}[i]\right)$.

If a dominating set $D$ induces a complete graph, then, $D$ is called clique dominating set, the clique domination number is the cardinality of a minimum clique dominating set, and is denoted by $\gamma_{c l}(G)$, if every vertex in $D$ is adjacent to another vertex in $D$, then $D$ is called total dominating set. The minimum cardinality of a total dominating set is called total domination number and is denoted by $\gamma_{t}(G)$. For any graph $G, \gamma(G) \leq \gamma_{t}(G) \leq \gamma_{c l}(G)$. Since the suggested dominating set, $D$, for $\Gamma\left(\mathbb{Z}_{n}[i]\right)$ in the second proof of Theorem 8.2 induces a complete graph, then $\gamma\left(\Gamma\left(\mathbb{Z}_{n}[i]\right)\right)=\gamma_{t}\left(\Gamma\left(\mathbb{Z}_{n}[i]\right)\right)=\gamma_{c l}\left(\Gamma\left(\mathbb{Z}_{n}[i]\right)\right)$.

\section{The Domination Number of $L\left(\Gamma\left(\mathbb{Z}_{n}[i]\right)\right)$}

In this section we determine the domination number of $L\left(\Gamma\left(\mathbb{Z}_{n}[i]\right)\right)$ when $n=t^{m}$ and $t$ is prime.

The study of the domination number of the line graph of $G$ leads to the study of edge or line domination number of $G$, that is, $\gamma(L(G))=\gamma^{\prime}(G)$. On the other hand, for any graph $G$, $\gamma_{i}^{\prime}(G)=\gamma^{\prime}(G)$, [17]. Further, if $G$ is the complete bipartite graph $K_{r, s}$, then $\gamma^{\prime}(G)=\min (r, s)$, thus we have the following.

Lemma 9.1. (i) $\gamma\left(L\left(\Gamma\left(\mathbb{Z}_{p}[i]\right)\right)\right)=\gamma_{i}^{\prime}\left(\Gamma\left(\mathbb{Z}_{p}[i]\right)\right)=\gamma^{\prime}\left(\Gamma\left(\mathbb{Z}_{p}[i]\right)\right)=p-1$.

(ii) $\gamma\left(L\left(\Gamma\left(\mathbb{Z}_{q_{1} q_{2}}[i]\right)\right)\right)=\gamma_{i}^{\prime}\left(\Gamma\left(\mathbb{Z}_{q_{1} q_{2}}[i]\right)\right)=\gamma^{\prime}\left(\Gamma\left(\mathbb{Z}_{q_{1} q_{2}}[i]\right)\right)=q_{1}$, where $q_{1}<q_{2}$.

Now, we study the domination number of the line graph of $\Gamma\left(\mathbb{Z}_{n}[i]\right)$ when $n$ is a power of a prime. The first theorem treats the case $n=2^{m}, m \geq 2$. Here we make use of the fact that $\Gamma\left(\mathbb{Z}_{2^{m}}[i]\right) \cong \Gamma\left(\mathbb{Z}_{2^{2 m}}\right)$, [12].

Theorem 9.2. For $n=2^{m}, m \geq 2$,

$$
\gamma\left(L\left(\Gamma\left(\mathbb{Z}_{n}[i]\right)\right)\right)=\gamma_{i}^{\prime}\left(\Gamma\left(\mathbb{Z}_{n}[i]\right)\right)=\gamma^{\prime}\left(\Gamma\left(\mathbb{Z}_{n}[i]\right)\right)=\left\lfloor\frac{1}{2}\left(2^{m}-1\right)\right\rfloor
$$

Proof. For $j=1,2, \ldots, 2 m-1$, let $A_{j}=\left\{\alpha 2^{2 m-j}: \alpha \in\left\{1,3, \ldots, 2^{j}-1\right\}\right\}$. Note that the sets $A_{j}$ form a partition to the vertices of $\Gamma\left(\mathbb{Z}_{2^{2 m}}\right)$. Let $S=\bigcup_{j=1}^{m} A_{j}$ and $T=\bigcup_{j=m+1}^{2 m-1} A_{j}$. Then the set $S$ induces a complete subgraph of $\Gamma\left(\mathbb{Z}_{2^{2 m}}\right)$ and the set $T$ form an independent set of it. And each vertex in $A_{k}$ is adjacent to each vertex in $\bigcup_{j=1}^{2 m-k} A_{j}$. $\Gamma\left(\mathbb{Z}_{2^{2 m}}\right)$ has no other edges. Let $D \subset E\left(\Gamma\left(\mathbb{Z}_{2^{2 m}}\right)\right)$ be a dominating set of vertices for $L\left(\Gamma\left(\mathbb{Z}_{2^{2 m}}\right)\right)$ with minimum cardinality. Since, the set $S$ induces a complete subgraph of $\Gamma\left(\mathbb{Z}_{2^{2 m}}\right)$ of order $2^{m}-1$, then $\gamma\left(L\left(\Gamma\left(\mathbb{Z}_{2^{m}}[i]\right)\right)\right) \geq\left\lfloor(1 / 2)\left(2^{m}-1\right)\right\rfloor$. On the other hand, since $D$ dominates all edges in the complete graph $\langle S\rangle, D$ also dominates every edge joining $S$ to $T$, recall that $T$ forms an independent set and so $\gamma\left(L\left(\Gamma\left(\mathbb{Z}_{2^{m}}[i]\right)\right)\right)=$ $\left\lfloor(1 / 2)\left(2^{m}-1\right)\right\rfloor$. 
The proof of Theorem 9.2. shows the set $T$ is an independent set with maximum cardinality in $\Gamma\left(\mathbb{Z}_{2^{m}}[i]\right)$, while the set $S$ induces a complete subgraph with maximum order.

So, the following corollary is obtained.

Corollary 9.3. For $n=2^{m}, m \geq 2$,

(i) $\omega\left(\Gamma\left(\mathbb{Z}_{n}[i]\right)\right)=2^{m}-1$,

(ii) $\beta\left(\Gamma\left(\mathbb{Z}_{n}[i]\right)\right)=2^{m}\left(2^{m-1}-1\right)$.

As another consequence to the proof of the preceding theorem, the following corollary, which gives the degree sequence for $\Gamma\left(\mathbb{Z}_{2^{m}}[i]\right)$, is obtained.

Corollary 9.4. For $j=1,2, \ldots, 2 m-1$, the graph $\Gamma\left(\mathbb{Z}_{2^{m}}[i]\right)$ has exactly $2^{j-1}$ vertices of degree $2^{2 m-j}-2$ if $1 \leq j \leq m$ and $2^{j-1}$ vertices of degree $2^{2 m-j}-1$ if $m+1 \leq j \leq 2 m-1$.

Proof. For each $v \in A_{j}$, where $1 \leq j \leq m, v^{2}=0$, so $\operatorname{deg}(v)=\left|\bigcup_{k=1}^{2 m-j} A_{k}\right|-1=2^{2 m-j}-2$. And for each $v \in A_{k}$, where $m+1 \leq k \leq 2 m-1, v^{2} \neq 0$, $\operatorname{sodeg}(v)=\left|\bigcup_{k=1}^{2 m-j} A_{k}\right|=2^{2 m-j}-1$.

Furthermore, The proof of the above theorem shows that the eccentricity of $2^{2 m-1}$ is 1 and the eccentricity of any other vertex in $\Gamma\left(\mathbb{Z}_{2^{2 m}}\right)$ is 2 , since the vertex 2 is adjacent only to the vertex $2^{2 m-1}$, and for any $x \in V\left(\Gamma\left(\mathbb{Z}_{2^{m}}[i]\right)\right), 2-2^{2 m-1}-x$, is a path of length 2 . This leads to the following corollary.

Corollary 9.5. The center of the graph $\Gamma\left(\mathbb{Z}_{2^{m}}[i]\right)$ is the set $\left\{2^{m-1}(1+i)\right\}$. $m \geq 2$.

Next, we we find the domination number of the line graph $L\left(\Gamma\left(\mathbb{Z}_{n}[i]\right)\right)$ when $n=q^{m}$,

Lemma 9.6. (i) For $m \geq 2$,

(1) If $A_{k j}=\left\{a q^{k}+b q^{j} i: a \in U\left(\mathbb{Z}_{q^{m-k}}\right), b \in U\left(\mathbb{Z}_{q^{m-j}}\right)\right\}$, then $\left|A_{k j}\right|=(q-1)^{2} q^{2 m-k-j-2}$ when $1 \leq k, j \leq m-1,\left|A_{m j}\right|=q^{m-j}-q^{m-j-1}$ and $\left|A_{k m}\right|=q^{m-k}-q^{m-k-1}$ when $k, j \neq m$,

(2) If $S=\left(\bigcup_{[m / 2] \leq k, j \leq m} A_{k j}\right)-A_{m m}$, then $|S|=q^{2\lfloor m / 2\rfloor}-1$.

(ii) For $m \geq 3$, if $T=\bigcup_{1 \leq k, j \leq[m / 2]-1} A_{k j}$, then $|T|=q^{2\lfloor m / 2\rfloor}\left(q^{[m / 2\rceil}-1\right)^{2}$.

Theorem 9.7. If $n=q^{m}, m \geq 2$, then $\gamma\left(L\left(\Gamma\left(\mathbb{Z}_{n}[i]\right)\right)\right)=\gamma^{\prime}\left(\Gamma\left(\mathbb{Z}_{n}[i]\right)\right)=\gamma_{i}^{\prime}\left(\Gamma\left(\mathbb{Z}_{n}[i]\right)\right)=(1 / 2)\left(q^{m}-\right.$ 1) if $m$ is even and $(1 / 2)\left(q^{2\lfloor m / 2\rfloor}+1\right)$ if $m$ is odd.

Proof. Let $A_{k j}, S$, and $T$ be defined as given in Lemma 9.6. Clearly, the set $S$ induces a complete subgraph of $\Gamma\left(\mathbb{Z}_{n}[i]\right)$ with maximum order if $m$ is even and $S \cup\left\{q^{\lfloor m / 2\rfloor}\right\}$ induces a complete subgraph of $\Gamma\left(\mathbb{Z}_{n}[i]\right)$ with maximum order if $m$ is odd. On other hand if $m \geq 3$, then $T$ form an independent set with maximum cardinality. Moreover, if a vertex $v$ belongs to the set $A_{r s}$, then $v$ is adjacent to every element in $A_{k j}$ where $m-\min \{r, s\} \leq k, j \leq m$ and $k, j \neq m$ at the same time. $\Gamma\left(\mathbb{Z}_{n}[i]\right)$ has no other edges.

As a consequence of the proof of Theorem 9.7, we conclude the following. 
Corollary 9.8. If $n=q^{m}, m \geq 2$, then

(i) $\omega\left(\Gamma\left(\mathbb{Z}_{n}[i]\right)\right)=q^{m}-1$ if $m$ is even and $q^{2\lfloor m / 2\rfloor}$ if $m$ is odd,

(ii) $\beta\left(L\left(\Gamma\left(\mathbb{Z}_{n}[i]\right)\right)\right)=1$ if $m=2$ and $\beta\left(L\left(\Gamma\left(\mathbb{Z}_{n}[i]\right)\right)\right)=q^{2\lfloor m / 2\rfloor}\left(q^{[m / 2]}-1\right)^{2}$ if $m \geq 3$.

Corollary 9.9. Let $n=q^{m}, m \geq 2$, and $v=a q^{r}+b q^{s} i$ where $a, b \in U\left(\mathbb{Z}_{n}\right)$. Then

$$
\operatorname{deg}(v)= \begin{cases}q^{2 \min \{r, s\}}-2, & \text { if } r, s \geq\left\lceil\frac{m}{2}\right\rceil, \\ q^{2 \min \{r, s\}}-1, & \text { if } r \text { or } s<\left\lceil\frac{m}{2}\right\rceil .\end{cases}
$$

Corollary 9.10. Let $n=q^{m}, m \geq 2$. Then

(i) the eccentricity of each $v \in A_{(m-1)(m-1)}$ is 1 and the eccentricity of any other vertex $v \in$ $\Gamma\left(\mathbb{Z}_{n}[i]\right)$ is 2 ,

(ii) the center of the graph $\Gamma\left(\mathbb{Z}_{n}[i]\right)$ is the set $A_{(m-1)(m-1)}$,

(iii) the radius of the graph $\Gamma\left(\mathbb{Z}_{n}[i]\right)$ equals 1 ,

(iv) the diameter of the graph $\Gamma\left(\mathbb{Z}_{n}[i]\right)$ equals 2 , for $m \geq 3$. $m \geq 2$.

Finally, we find the domination number of the line graph $L\left(\Gamma\left(\mathbb{Z}_{n}[i]\right)\right)$ when $n=p^{m}$,

Recall that $\mathbb{Z}_{p^{m}}[i] \cong \mathbb{Z}_{p^{m}} \times \mathbb{Z}_{p^{m}}$. Let, $A_{k j}=\left\{\left(a p^{k}, b p^{j}\right): a \in U\left(\mathbb{Z}_{p^{m-k}}\right), b \in U\left(\mathbb{Z}_{p^{m-j}}\right)\right\}$. Clearly, the sets $A_{k j}, 0 \leq k, j \leq m$ and not both $k, j=m$ or 0 , partition the vertices of $\Gamma\left(\mathbb{Z}_{p^{m}} \times\right.$ $\left.\mathbb{Z}_{p^{m}}\right)$.

Lemma 9.11. (i) For $m \geq 2$ :

(1) if $S=\left(\bigcup_{[m / 2] \leq k, j \leq m} A_{k j}\right)-A_{m m}$, then $s=|S|=p^{2\lfloor m / 2\rfloor}-1$,

(2) if $L_{1}=\bigcup_{0 \leq k \leq[m / 2]-1} A_{k m}$ and $L_{2}=\bigcup_{0 \leq k \leq[m / 2]-1} A_{m k}$, then $l=\left|L_{1}\right|=\left|L_{2}\right|=p^{m}-p^{\lfloor m / 2\rfloor}$,

(ii) for $m \geq 3$ :

(1) if $B=\bigcup_{k=1}^{[m / 2]-1} \bigcup_{j=m-k}^{m-1} A_{k j}$, then $b=|B|=\left(p^{m}-p^{m-1}\right)([m / 2\rceil-1)-\left(p^{m-1}-p^{\mid m / 2]}\right)$,

(2) if $T=\bigcup_{0 \leq k, j \leq\lceil m / 2]-1} A_{k j}-A_{0,0}$, then $t=|T|=\left(p^{m-1}-p^{\lfloor m / 2\rfloor}\right)^{2}+2(p-1)\left(p^{2 m-2}-\right.$ $\left.p^{2 m-[m / 2]-1}\right)$,

(iii) for $m \geq 4$ :

If $W_{1}=\bigcup_{k=[m / 2]}^{m-1} \bigcup_{j=1}^{m-k-1} A_{k j}, W_{2}=\bigcup_{j=[m / 2]}^{m-1} \bigcup_{k=1}^{m-j-1} A_{k j}$ and $W=W_{1} \cup W_{2}$, then $w=$ $|W|=2 p^{m-1}\left(\left(p^{\lfloor m / 2\rfloor}-1\right)-\lfloor m / 2\rfloor(p-1)\right)$.

Theorem 9.12. Let $n=p^{m}, m \geq 2$ and $s, l$ and $b$ be defined as given in Lemma 9.11, then $\gamma\left(L\left(\Gamma\left(\mathbb{Z}_{n}[i]\right)\right)\right)=\gamma^{\prime}\left(\Gamma\left(\mathbb{Z}_{n}[i]\right)\right)=\gamma_{i}^{\prime}\left(\Gamma\left(\mathbb{Z}_{n}[i]\right)\right)=(s / 2)+l+b$ if $m$ is even and $(s / 2)+l+b+1$ if $m$ is odd.

Proof. Using the same notations of Lemma 9.11. Note that the set $S$ induces a complete subgraph of $\Gamma\left(\mathbb{Z}_{n}[i]\right), K_{s}$. Thus, any edge dominating set for $\Gamma\left(\mathbb{Z}_{p^{m}} \times \mathbb{Z}_{p^{m}}\right)$ must contain $s / 2$ edges to dominate $K_{s}$. If $m \geq 3$, the set $L=L_{1} \cup L_{2}$ induces a complete bipartite graph $K_{l, l}$ with bipartite sets $L_{1}$ and $L_{2}$. This contributes $l$ edges in the dominating edge set for $\Gamma\left(\mathbb{Z}_{p^{m}} \times \mathbb{Z}_{p^{m}}\right)$. 
Edges joining vertices in $K_{l, l}$ to vertices in $K_{s}$ are covered by the same edge dominating sets for $K_{l, l}$ and $K_{s}$. Moreover, vertices in $A_{k 0}$ and $A_{0 k}$, where $1 \leq k \leq m-1$, are only adjacent to some vertices in $K_{s}$ and $K_{l, l}$.

On the other hand, if $m \geq 3$, the set $T$ is an independent set. Fortunately, vertices in $T$ are only adjacent to vertices in $S$. So, any edge dominating set for $K_{S}$ also dominates edges between $S$ and $T$.

Now, for each $1 \leq k \leq[m / 2]-1$, and $m-k \leq j \leq m$, the set $A_{k j} \cup A_{j k}$ induces a complete bipartite graph with bipartite sets $A_{k j}$ and $A_{j k}$. In order to dominate this collection of complete bipartite graphs induced by $A_{k j} \cup A_{j k}$ we need $b$ edges in the edge dominating set for $\Gamma\left(\mathbb{Z}_{p^{m}} \times \mathbb{Z}_{p^{m}}\right)$. Fortunately, this dominating set with $b$ elements also dominates all edges in $E\left(\Gamma\left(\mathbb{Z}_{p^{m}} \times \mathbb{Z}_{p^{m}}\right)\right)$ which are incident to any edge in this collection.

Finally, observe that if $m \geq 4$, then vertices in $W$ are only adjacent to some vertices in $K_{s}$ as well as in the collection of the complete bipartite graphs. The graph $\Gamma\left(\mathbb{Z}_{p^{m}} \times \mathbb{Z}_{p^{m}}\right)$ has no other edges.

The above proof shows that $S$ induces a complete graph in $\Gamma\left(\mathbb{Z}_{p^{m}} \times \mathbb{Z}_{p^{m}}\right)$. In fact, $K_{s}$ is a complete subgraph with maximum order in case $m$ is even, while if $m$ is odd we can add one additional vertex of some $A_{k j}$, where either $k$ or $j$, say $k$, is $\lfloor m / 2\rfloor$ while $j$ is greater than $\lfloor m / 2\rfloor$. On the other hand, the set $T \cup W \cup \bigcup_{k=[m / 2]}^{m-1}\left(A_{k, 0} \cup A_{0, k}\right) \cup A_{m, 0}$ is a maximum independent set of order $t+w+r$, where $r=\left|\bigcup_{k=[m / 2]}^{m-1}\left(A_{k, 0} \cup A_{0, k}\right) \cup A_{m, 0}\right|=p^{m-1}(p-1)\left(2 p^{\lfloor m / 2\rfloor}-1\right)$.

Thus, using the same notation of Lemma 9.11 and the proof of the above theorem, we obtain the following corollary.

Corollary 9.13. If $n=p^{m}$, then

(i) $\omega\left(\Gamma\left(\mathbb{Z}_{n}[i]\right)\right)=s$ if $m$ is even and $s+1$ if $m$ is odd, for $m \geq 2$,

(ii) $\beta\left(L\left(\Gamma\left(\mathbb{Z}_{n}[i]\right)\right)\right)=r$, if $m=2, \beta\left(L\left(\Gamma\left(\mathbb{Z}_{n}[i]\right)\right)\right)=r+t$, if $m=3$, and $\beta\left(L\left(\Gamma\left(\mathbb{Z}_{n}[i]\right)\right)\right)=$ $r+t+w$, for $m \geq 3$.

Corollary 9.14. If $n=p^{m}, m \geq 2$, then

(i) $E=\left\{v \in V\left(\Gamma\left(\mathbb{Z}_{n}[i]\right)\right): v=(u, w)\right.$ where either $u$ or $\left.w \in U\left(\mathbb{Z}_{p^{m}}\right)\right\}$ has eccentricity 3 , while all other vertices has eccentricity 2 ,

(ii) the center of the graph $\Gamma\left(\mathbb{Z}_{n}[i]\right)$ is the set $C=\left\{v \in V\left(\Gamma\left(\mathbb{Z}_{n}[i]\right)\right): v=(u, w)\right.$, where both $u$ and $\left.w \in Z\left(\mathbb{Z}_{p^{m}}\right)\right\}-\{(0,0)\}$,

(iii) the radius of the graph $\Gamma\left(\mathbb{Z}_{n}[i]\right)$ equals 2 ,

(iv) the diameter of the graph $\Gamma\left(\mathbb{Z}_{n}[i]\right)$ equals 3.

Proof. (i) First, note that $\Gamma\left(\mathbb{Z}_{p^{m}}[i]\right)$ has no vertex of eccentricity 1 , otherwise $\gamma\left(\Gamma\left(\mathbb{Z}_{p^{m}}[i]\right)\right)=1$. Let $u, w, \alpha, \beta \in U\left(\left(\mathbb{Z}_{p^{m}}\right)\right)$ and $1 \leq r, s \leq m-1$. If $(x, y)$ is adjacent to both $\left(u p^{r}, \beta\right)$ and $\left(\alpha, w p^{r}\right)$, then $x=y=0$. So, $d\left(\left(u p^{r}, \beta\right),\left(\alpha, w p^{s}\right)\right)=3$, and hence, the eccentricity of each vertex in $E$ is 3 . If $\left(u p^{r}, y\right),\left(w p^{s}, x\right)$ are nonadjacent, then $\left(p^{m-1}, 0\right)$ is adjacent to both vertices. Similarly, if $\left(x, u p^{r}\right),\left(y, w p^{s}\right)$ are nonadjacent, then $\left(0, p^{m-1}\right)$ is adjacent to both vertices.

\section{References}

[1] I. Beck, “Coloring of commutative rings," Journal of Algebra, vol. 116, no. 1, pp. 208-226, 1988.

[2] D. F. Anderson and P. S. Livingston, "The zero-divisor graph of a commutative ring," Journal of Algebra, vol. 217, no. 2, pp. 434-447, 1999. 
[3] D. F. Anderson, A. Frazier, A. Lauve, and P. S. Livingston, "The zero-divisor graph of a commutative ring. II," in Ideal Theoretic Methods in Commutative Algebra, vol. 220 of Lecture Notes in Pure and Applied Mathematics, pp. 61-72, Marcel Dekker, New York, NY, USA, 2001.

[4] S. Akbari and A. Mohammadian, "On the zero-divisor graph of a commutative ring," Journal of Atgebra, vol. 274, no. 2, pp. 847-855, 2004.

[5] D. F. Anderson and A. Badawi, "On the zero-divisor graph of a ring," Communications in Algebra, vol. 36, no. 8, pp. 3073-3092, 2008.

[6] N. Cordova, C. Gholston, and H. Hauser, The Structure of Zero-Divisor Graphs, Summer Undergraduate Mathematical Sciences Research Institute, Miami University, 2005.

[7] A. Duane, "Proper Coloring and p-partite structures of the zero-divisor graph," Rose-Hulman Undergraduate Mathematics Journal, vol. 7, no. 2, pp. 1-7, 2006.

[8] V. K. Bhat, R. Raina, N. Nehra, and O. Prakash, "A note on zero divisor graph over rings," International Journal of Contemporary Mathematical Sciences, vol. 2, no. 13-16, pp. 667-671, 2007.

[9] P. F. Lee, Line graph of zero divisor graph in commutative rings, M.S. thesis, Colorado Christian University, 2007.

[10] A. Phillips, J. Rogrers, K. Tolliver, and F. Worek, Uncharted Territory of Zero-Divisor Graphs and Their Complements, Summer Undergraduate Mathematical Science Research Institute, Miami University, 2004.

[11] E. Abu Osba, S. Al-Addasi, and N. Abu Jaradeh, "Zero divisor graph for the ring of Gaussian integers modulo n," Communications in Algebra, vol. 36, no. 10, pp. 3865-3877, 2008.

[12] E. Abu Osba, S. Al-Addasi, and B. Al-Khamaiseh, "Some properties of the zero-divisor graph for the ring of Gaussian integers modulo n," Glasgow Mathematical Journal, vol. 53, no. 2, pp. 391-399, 2011.

[13] H. J. Veldman, "A result on Hamiltonian line graphs involving restrictions on induced subgraphs," Journal of Graph Theory, vol. 12, no. 3, pp. 413-420, 1988.

[14] S. Skiena, Implementing Discrete Mathematics: Combinatorics and Graph Theory with Mathematica, Addison-Wesley, Redwood City, Calif, USA, 1990.

[15] J. Sedláček, "Some properties of interchange graphs," in Theory of Graphs and its Application, pp. 145150, Academic Press, New York, NY, USA, 1962.

[16] S. P. Redmond, "Central sets and radii of the zero-divisor graphs of commutative rings," Communications in Algebra, vol. 34, no. 7, pp. 2389-2401, 2006.

[17] S. Arumugam and S. Velammal, "Edge domination in graphs," Taiwanese Journal of Mathematics, vol. 2, no. 2, pp. 173-179, 1998. 


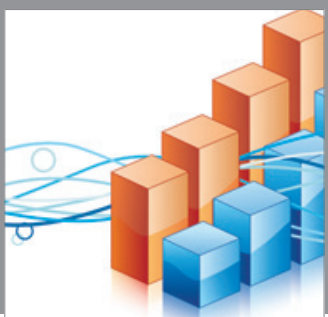

Advances in

Operations Research

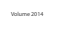

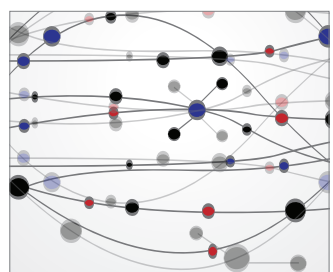

\section{The Scientific} World Journal
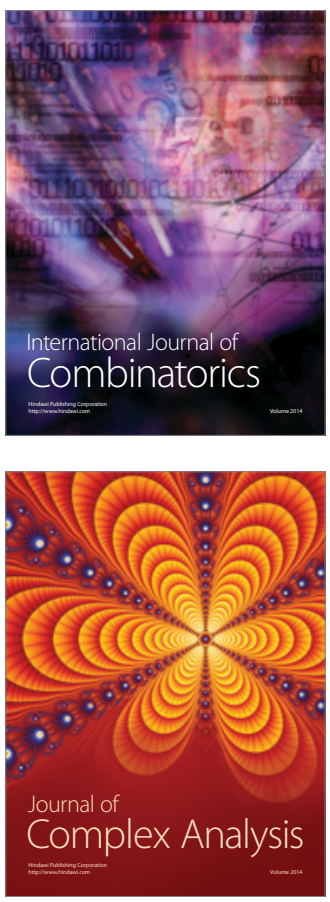

International Journal of

Mathematics and

Mathematical

Sciences
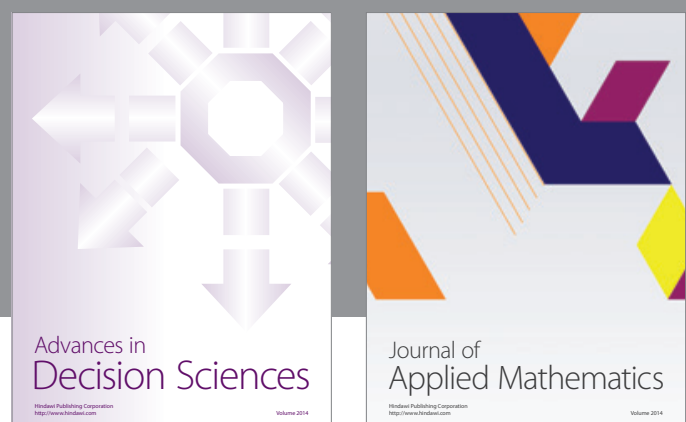

Journal of

Applied Mathematics
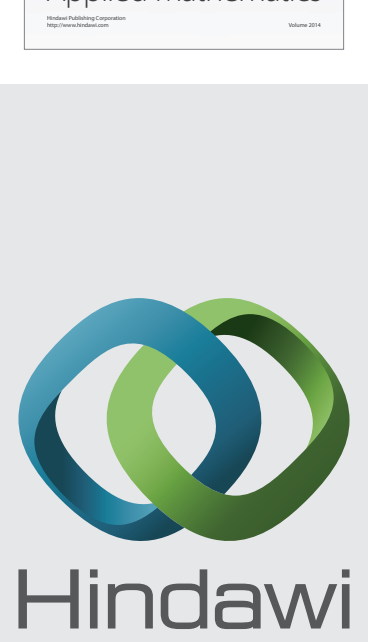

Submit your manuscripts at http://www.hindawi.com
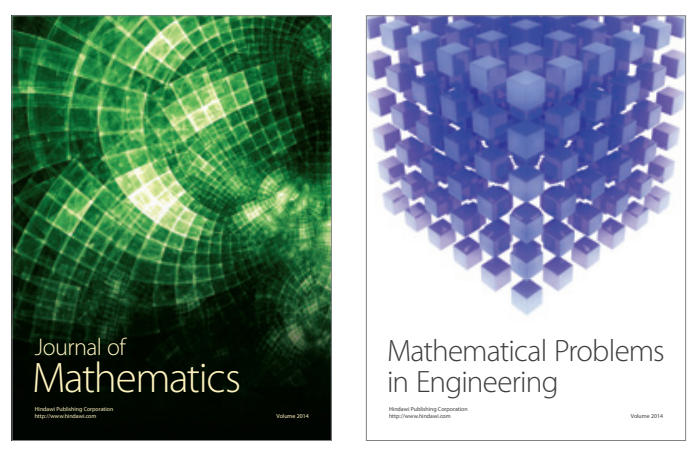

Mathematical Problems in Engineering
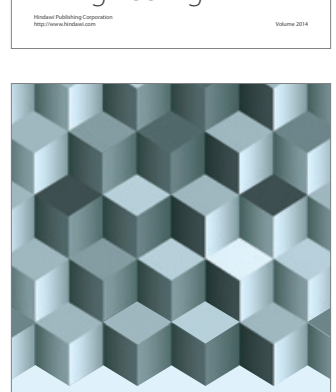

Journal of

Function Spaces
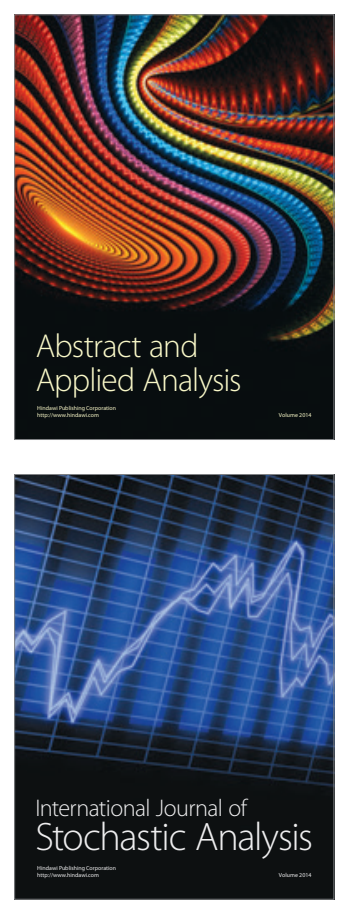

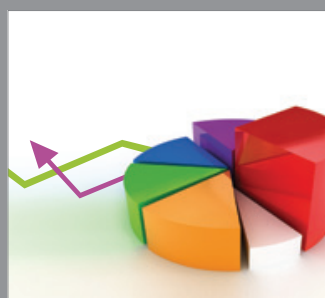

ournal of

Probability and Statistics

Promensencen
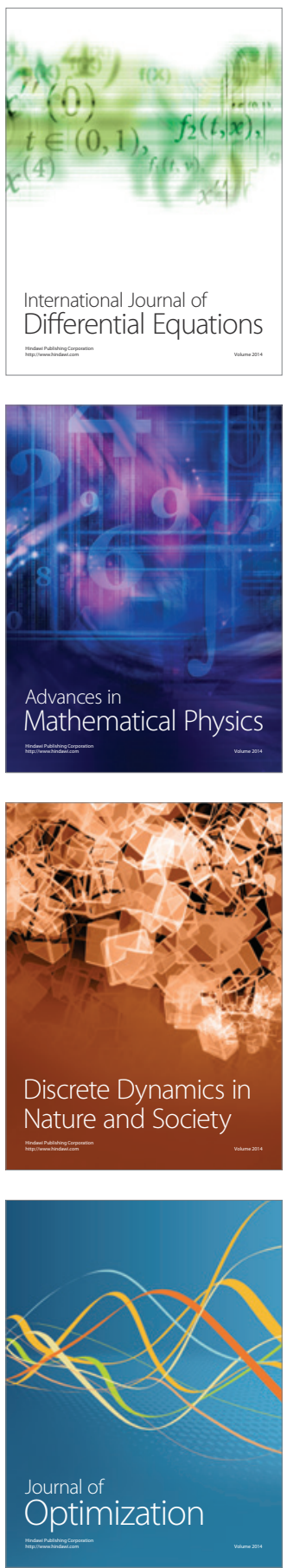